\title{
Coincidence theorems and minimax inequalities in abstract convex spaces
}

Yeol Je Cho' ${ }^{1}$ Mohsen Rostamian Delavar ${ }^{2}$, Seyed Asghar Mohammadzadeh ${ }^{3}$ and Mehdi Roohi ${ }^{4^{*}}$

* Correspondence: m.roohi@gu.ac.ir ${ }^{4}$ Department of Mathematics, Faculty of Sciences Golestan University, P.O.Box. 155, Gorgan, Iran

Full list of author information is available at the end of the article

\section{Abstract}

In this paper, we deal with the notion of abstract convex spaces via minimal spaces as an extended version of other forms of convexity and establish some well-known results such as coincidence theorems for the classes $m$-KKM and ms-KKM of multimaps and Ky Fan's type minimax inequality.

Mathematics Subject Classification (2000): 26A51; 26B25; 54H25; 55M20; 47H10; 54A05.

Keywords: abstract convexity, abstract convex space, KKM theorem, coincidence theorem, minimax inequality

\section{Introduction}

Many problems in nonlinear analysis can be solved by showing the nonemptyness of the intersection of certain family of subsets of an underlying set. Each point of the intersection can be a fixed point, a coincidence point, an equilibrium point, a saddle point and an optimal point or others of the corresponding equilibrium problem under consideration.

The first remarkable result on the nonempty intersection was the celebrated KnasterKuratowski-Mazurkiewicz theorem (simply, KKM principle) in 1929 [1], which concerns with certain types of multimaps called the KKM maps later. The KKM theory first called by Park in [2] and [3] was the study of KKM maps and their applications. At the beginning, the theory was mainly devoted to study on convex subsets of topological vector spaces. Later, it has been extended to convex spaces by Lassonde [4], to $C$-spaces (or $H$-spaces) by Horvath [5-8] and to others.

In 1993, Park and Kim [9] introduced the concept of generalized convex spaces, and the KKM theory is extended to generalized convex (G-convex) in a sequence of papers by many authors (for details, see [2,3,9] and [10]) and the references cited therein. Note that, in the KKM theory, there have appeared a number of coincidence theorems with many significant applications.

In 1996, since Chang and Yen [11] introduced the class $\operatorname{KKM}(X, Y)$ of multimaps, it was developed by many authors. Recently, Lin et al. [12] studied the class $\operatorname{KKM}(X, Y)$ in topological vector space and proved some KKM type coincidence and fixed point theorems. In all KKM type theorems, the convexity plays important role and so many efforts have done to establish KKM type theorems without convexity structure. For

(C) 2011 Je Cho et al; licensee Springer. This is an Open Access article distributed under the terms of the Creative Commons Attribution License (http://creativecommons.org/licenses/by/2.0), which permits unrestricted use, distribution, and reproduction in any medium, provided the original work is properly cited. 
example, see KKM type theorems in minimal generalized convex spaces [13-15], G-convex spaces [9], FC-spaces [16] and topological ordered spaces [17].

Recently, Zafarani [18] and Park [19] have introduced a new concept of abstract convex space and certain broad classes $\mathrm{KC}$ and $\mathrm{KO}$ of multimaps (having the KKM property). With this new concept, the KKM type maps were used to obtain matching theorems, coincidence theorems, fixed point theorems and others.

In this paper, by using the concept of abstract convexity and minimal spaces, the classes $m-\operatorname{KKM}(X, Y)$ and $m s-\operatorname{KKM}(X, Y, Z)$ as a generalization of $\operatorname{KKM}(X, Y)$ and $s$-KKM $(X, Y, Z)$ are introduced. Some generalized KKM and $s$-KKM type theorems for minimal transfer closed valued multimaps are established. As applications, some new coincidence theorems and a new version of Ky Fan's minimax theorem are obtained.

\section{Preliminaries}

A multimap $F: X \rightarrow Y$ is a function from a set $X$ into the power set of $Y$. For any $A \subseteq X$, set $F(A)=\cup_{x \in A} F(x)$. Define the graph of $F$ as $G_{F}=\{(x, y) \in X \times Y: y \in F(x)\}$. Furthermore, for any $x \in X$, define $F^{c}(x)=\{y \in Y: y \notin F(x)\}$ and, for any $y \in Y, F^{*}(y)=\{x \in X: y \in F(x)\}$ and $F^{*}(y)=\{x \in X: y \notin F(x)\}$. Note that $F^{*}(y)=X \backslash F^{-}(y)$ for any $y \in Y$. For any set $D$, put $\langle D\rangle$ as the family of all nonempty finite subsets of $D$.

Proposition 2.1. [20]Suppose $F, G: X \rightarrow Y$ are two multimaps. Then we have the following.

(1) for each $x \in X, F(x) \subseteq G(x)$ if and only if $G^{*}(y) \subseteq F^{*}(y)$ for each $y \in Y$,

(2) $y \notin F(x)$ if and only if $x \in F^{*}(y)$,

(3) for each $x \in X,\left(F^{*}\right)^{*}(x)=F(x)$,

(4) for each $x \in X, F(x) \neq \emptyset$ if and only if $\bigcap_{y \in Y} F^{*}(y)=\emptyset$,

(5) for each $y \in Y,\left(F^{c}\right)^{*}(y)=F^{*}(y)$,

(6) for each $y \in Y,\left(F^{*}\right)^{c}(y)=F^{*}(y)$.

A family $\mathcal{M} \subseteq \mathcal{P}(X)$ is said to be a minimal structure on, $\mathrm{X}$ if $\emptyset, X \in \mathcal{M}$. In this case, $(X, \mathcal{M})$ is called a minimal space. For example, let $(X, \tau)$ be a topological space, then $\tau, S O(X), P O(X), \alpha O(X)$ and $\beta O(X)$ are minimal structures on $X$ [21]. In a minimal space $(X, \mathcal{M}), A \in \mathcal{P}(X)$ is called an m-open set if $A \in \mathcal{M}$ and also $B \in \mathcal{P}(X)$ is called an $m$-closed set if $B^{c} \in \mathcal{M}$. Set $m-\operatorname{Int}(A)=\cup\{U: U \subseteq A, U \in \mathcal{M}\}$ and $m-\mathrm{Cl}(A)=\cap\left\{B: A \subseteq B, B^{c} \in \mathcal{M}\right\}$. Notice that, for any set $A \subseteq X, m-\mathrm{Cl}(A)$ (resp., $m$-Int $(A))$ is not necessarily $m$-closed (resp., $m$-open).

It is not hard to see that there are many minimal spaces which are not topological space. Furthermore, in the following example, it is shown that there are some linear minimal spaces which are not topological vector space. Moreover in [13], there is a minimal G-convex space which is not $G$-convex space (see [9] and [13]).

Definition 2.2[22]. Let $(X, \mathcal{M})$ and $(Y, \mathcal{N})$ be two minimal spaces. A function $f:(X, \mathcal{M}) \rightarrow(Y, \mathcal{N})$ is called minimal continuous (briefly $m$-continuous) if $f^{-1}(U) \in \mathcal{M}$ for any $U \in \mathcal{N}$.

Example 2.3[13]. Consider the real field $\mathbb{R}$. Clearly $\mathcal{M}=\{(a, b): a, b \in \mathbb{R} \cup\{ \pm \infty\}\}$ is a minimal structure on $\mathbb{R}$. We claim that $\mathcal{M}$ is a linear minimal structure on $\mathbb{R}$. For this, we must prove that, two operations + and $\cdot$ are $m$-continuous. Suppose $\left(x_{0}, y_{0}\right) \in+{ }^{-1}(a, b)$ 
and so $x_{0}+y_{0} \in(a, b)$. Put $\varepsilon=\min \left\{x_{0}+y_{0}-a, b-\left(x_{0}+y_{0}\right)\right\}$ and so $x_{0} \in\left(x_{0}-\frac{\varepsilon}{2}, x_{0}+\frac{\varepsilon}{2}\right)$ and $y_{0} \in\left(y_{0}-\frac{\varepsilon}{2}, y_{0}+\frac{\varepsilon}{2}\right)$. Hence,

$$
x_{0}+y_{0} \in\left(\left(x_{0}-\frac{\varepsilon}{2}, x_{0}+\frac{\varepsilon}{2}\right)+\left(y_{0}-\frac{\varepsilon}{2}, y_{0}+\frac{\varepsilon}{2}\right)\right) \subseteq(a, b) ;
$$

which implies that $+^{-1}(a, b)$ is $m$-open in the minimal product space $\mathbb{R} \times \mathbb{R}$; that is + is $m$-continuous. Also, suppose $\left(\alpha_{0}, x_{0}\right) \in \cdot^{-1}(a, b)$. Since $\alpha_{0} x_{0} \in(a, b)$ and $\lim _{s, t \rightarrow 0}\left(\alpha_{0}-s\right)\left(x_{0}-\right.$ $t)=\alpha_{0} x_{0}$, so one can find some $0<\delta$ for which $\left|\alpha_{0}-s\right|<\delta$ and $\left|x_{0}-t\right|<\delta$ imply that $a<$ $\left(\alpha_{0}-s\right)\left(x_{0}-t\right)<b$. Therefore, $\left(\alpha_{0}, x_{0}\right) \in\left(\alpha_{0}-\delta, \alpha_{0}+\delta\right) \cdot\left(x_{0}-\delta, x_{0}+\delta\right) \subseteq(a, b)$; i.e., ${ }^{-1}(a, b)$ is $m$-open in the minimal product space $\mathbb{R} \times \mathbb{R}$, which implies that the operation $\cdot$ is $m$-continuous.

For the main results in this paper, we recall some basic definitions and results. More details can be found in [13,15,21-24] and [22] and references therein.

Proposition 2.4. [21] For any two sets $A$ and $B$,

(1) $m$-Int $(A) \subseteq A$ and $m-\operatorname{Int}(A)=A$ if $A$ is an m-open set.

(2) $A \subseteq m-\mathrm{Cl}(A)$ and $A=m-\mathrm{Cl}(A)$ if $A$ is an $m$-closed set.

(3) $m-\operatorname{Int}(A) \subseteq m-\operatorname{Int}(B)$ and $m-\mathrm{Cl}(A) \subseteq m-\mathrm{Cl}(B)$ if $A \subseteq B$.

(4) $m-\operatorname{Int}(m-\operatorname{Int}(A))=m-\operatorname{Int}(A)$ and $m-\mathrm{Cl}(m-\mathrm{Cl}(B))=m-\mathrm{Cl}(B)$,

(5) $(m-\mathrm{Cl}(A))^{c}=m-\operatorname{Int}\left(A^{c}\right)$ and $(m-\operatorname{Int}(A))^{c}=m-\mathrm{Cl}\left(A^{c}\right)$.

Definition 2.5[14]. Let $X$ be a nonempty set and $Y$ be a minimal space. A multimap $F: X \rightarrow Y$ is said to be

(1) minimal transfer open valued if, for each $x \in X$ and $y \in F(x)$, there exists $x_{0} \in$ $X$ such that $y \in m-\operatorname{Int}\left(F\left(x_{0}\right)\right)$.

(2) minimal transfer closed valued if, for any $x \in X$ and $y \notin F(x)$, there exists $x_{0} \in$ $X$ such that $y \notin m-\mathrm{Cl}\left(F\left(x_{0}\right)\right)$.

Theorem 2.6. Suppose that $X$ is a nonempty set and $Y$ is a minimal space. Then the following are equivalent.

(1) The multimap $F: X \rightarrow Y$ is minimal transfer closed valued,

(2) $n_{x \in X} F(x)=n_{x \in X} m-C l(F(x))$,

(3) $\cup_{x \in X} F^{c}(x)=\cup_{x \in X} m-\operatorname{Int}\left(F^{c}(x)\right)$,

(4) $\cup_{x \in X} F^{c}(x)=\cup_{x \in X}(m-\mathrm{Cl}(F(x)))^{c}$,

(5) $F^{c}$ is minimal transfer open valued.

Proof. See theorems 2.1, 2.2 and 2.3 in [14].

Definition 2.7[22]. For a minimal space $(X, \mathcal{M})$,

(1) a family $\mathcal{A}=\left\{A_{j}: j \in J\right\}$ of $m$-open sets in $X$ is called an m-open cover of $K$ if $K \subseteq \cup_{j \in J} A_{j}$. Any subfamily of $\mathcal{A}$ which is also an $m$-open cover of $K$ is called a subcover of $\mathcal{A}$ for $K$,

(2) a subset $K$ of $X$ is m-compact whenever given any $m$-open cover of $K$ has a finite subcover. 
Definition 2.8. Suppose that $X$ is a nonempty set and $Y$ is a minimal space. A multimap $T: X \rightarrow Y$ is called m-compact if $m-\mathrm{Cl}(T(X))$ is an $m$-compact subset of $Y$.

Lemma 2.9. [15] Suppose that $(X, \mathcal{M})$ is an m-compact minimal space and $\left\{A_{i}: i \in I\right\}$ is a family of subsets of $X$. If $\left\{m-\mathrm{Cl}\left(A_{i}\right): i \in I\right\}$ has the finite intersection property, then $\bigcap_{i \in I} m-\operatorname{Cl}\left(A_{i}\right) \neq \emptyset$.

\section{Coincidence theorems in abstract convex spaces}

Now, we give some definitions for the results in this section as follows.

Definition 3.1[19]. An abstract convex space $(X, D, \Gamma)$ consists of two nonempty sets $X, D$ and a multimap $\Gamma:\langle D\rangle \rightarrow X$. In case to emphasize $X \supseteq D,(X, D, \Gamma)$ will be denoted by $(X \supseteq D, \Gamma)$; and if $X=D$, then $(X \supseteq X ; \Gamma)$ by $(X, \Gamma)$. If $D \subseteq X$ and $E \subseteq X$, then $E$ is called abstract convex if $\Gamma(A) \subseteq E$ for each $A \in\langle D \cap E\rangle$. Obviously, for any $B \subseteq D$, we can define $\mathrm{Co}_{\Gamma}(B)=\mathrm{U}\left\{\Gamma_{A} \mid A \in\langle B\rangle\right\}$.

Motivated by the results of Chang et al. [23], we introduce a new definition about the family of multimaps with the $m s-\mathrm{KKMC}(m s-\mathrm{KKMO})$ property in minimal abstract convex space as follows.

Definition 3.2. Let $Y$ be a nonempty set, $Z$ be a minimal space, $s: Y \rightarrow D$ be a function and $(X, D, \Gamma)$ be an abstract convex space. Let $T: X \multimap Z$ and $F: Y \multimap Z$ be two multimaps. we say that $F$ is generalized $s$-KKM with respect to $T$ if

$$
T(\Gamma(s(A))) \subseteq F(A) \text { for any } A \in\langle Y\rangle .
$$

The multimap $T$ has $m s$-KKMC (resp. $m s-\mathrm{KKMO}$ ) property if the following conditions.

(1) for any $y \in D, F(y)=m-\mathrm{Cl}\left(A_{y}\right)\left(\right.$ resp. $\left.F(y)=m-\operatorname{Int}\left(A_{y}\right)\right)$ for some $A_{y} \subseteq Z$,

(2) $F$ is generalized $s$-KKM with respect to $T$,

imply that the family $\{F(y): y \in D\}$ has the finite intersection property.

Set

(1) $m s-\mathrm{KKMC}(X, Y, Z)=\{T: X \multimap Z: T$ has $m s-\mathrm{KKMC}$ property $\}$,

(2) $m s-\mathrm{KKMO}(X, Y, Z)=\{T: X \rightarrow Z: T$ has $m s-\mathrm{KKMO}$ property $\}$

Remark 3.3. Note that

(1) If $D=Y$ and the function $s: Y \rightarrow D$ is the identity map $\operatorname{Id}_{D}$, the class $m s$ $\operatorname{KKMC}(X, Y, Z)$ reduces to the class $m-\operatorname{KKMC}(X, Z)$ introduced and investigated in [13-15].

(2) The classes $m$ - $\operatorname{KKMC}(X, Y)$ and $m s-\operatorname{KKMC}(X, Y, Z)$ generalize the classes KKM $(X, Y)$ and $s-\operatorname{KKM}(X, Y, Z)$ in $G$-convex spaces and their subclasses (see [24]), in topological space.

Proposition 3.4. Suppose that $X$ is a minimal space and $A, B \subseteq X$ such that $B$ is $m$-compact and $m-\mathrm{Cl}(A) \subseteq B$. Then $m-\mathrm{Cl}(A)$ is m-compact.

Proof. Suppose that $m-\mathrm{Cl}(A) \subseteq \mathrm{U}_{i \in I} \mathrm{G}_{i}$ for any $m$-open cover $\left\{G_{i}: i \in I\right\}$. Since $B \subseteq m$-Cl $(A) \cup(m-\mathrm{Cl}(A))^{c}$, we have $B \subseteq m-\mathrm{Cl}(A) \cup m$-Int $\left(A^{c}\right)$. From the definition of " $m$-Int", it follows that 


$$
B \subseteq \bigcup_{i \in I} G_{i} \cup m-\operatorname{Int}\left(A^{c}\right) \subseteq \bigcup_{i \in I} G_{i} \cup \bigcup_{j \in J}\left\{U_{j}: U_{j} \in \mathcal{M}, U_{j} \subseteq A^{c}\right\}
$$

Thus the compactness of $B$ implies that

$$
B \subseteq \bigcup_{i=1}^{n} G_{i} \cup \bigcup_{j=1}^{m}\left\{U_{j}: U_{j} \in \mathcal{M}, U_{j} \subseteq A^{c}\right\} \subseteq \bigcup_{i=1}^{n} G_{i} \cup m-\operatorname{Int}\left(A^{c}\right)
$$

Therefore, we have $B \subseteq \bigcup_{i=1}^{n} G_{i} \cup(m-\mathrm{Cl}(A))^{c}$, which implies that $m-\mathrm{Cl}(A)=B \cap m-\mathrm{Cl}(A) \subseteq \bigcup_{i=1}^{n} G_{i}$. Hence $m-\mathrm{Cl}(A)$ is $m$-compact. This completes the proof.

Theorem 3.5. Let $(X, D, \Gamma)$ be an abstract convex space and $Z$ be a minimal space. Suppose that $s: Y \rightarrow D$ is a mapping, $T \in m s-\operatorname{KKMC}(X, Y, Z)$ is an m-compact mapping such that $F: Y \rightarrow Z$ is generalized s-KKM with respect to $T$. Then we have

$$
m-\operatorname{Cl}\left(T\left(\mathrm{Co}_{\Gamma}(s(Y))\right)\right) \cap \bigcap_{\gamma \in Y} m-\operatorname{Cl}(F(y)) \neq \emptyset .
$$

Proof. Consider the multimap $G: Y \rightarrow m-\mathrm{Cl}\left(T\left(\mathrm{Co}_{\Gamma}(s(Y))\right)\right)$ defined by

$$
\mathrm{G}(y)=m-\mathrm{Cl}\left(m-\mathrm{Cl}\left(T\left(\mathrm{Co}_{\Gamma}(s(Y))\right)\right) \cap F(y)\right)
$$

for any $y \in Y$. It is easy to check that $G$ is well defined. Since $F$ is generalized $s$-KKM with respect to $T$, for any $A \in\langle Y\rangle, T(\Gamma(s(A))) \subseteq F(A)$. Also, $T(\Gamma(s(A))) \subseteq m-\mathrm{Cl}\left(T\left(\mathrm{Co}_{\Gamma}(s\right.\right.$ $(Y))))$ for each $A \in\langle Y\rangle$. Hence $T(\Gamma(s(A))) \subseteq m-\mathrm{Cl}\left(m-\mathrm{Cl}\left(T\left(\mathrm{Co}_{\Gamma}(s(Y))\right)\right) \cap F(A)\right)=G(A)$ and so $G$ is generalized $s$-KKM with respect to $T$. Since $T \in m s-\operatorname{KKMC}(X, Y, Z)$, it follows that $\left\{G(y)=m-\mathrm{Cl}\left(m-\mathrm{Cl}\left(T\left(\mathrm{Co}_{\Gamma}(s(Y))\right)\right) \cap F(Y)\right): y \in Y\right\}$ has the finite intersection property in $m-\mathrm{Cl}\left(T\left(\mathrm{Co}_{\Gamma}(s(Y))\right)\right)$, which is an $m$-compact subset of $Z$ by Proposition 3.4. Thus it follows from Lemma 2.9 that $\bigcap_{y \in Y} m-\operatorname{Cl}\left(m-\operatorname{Cl}\left(T\left(\mathrm{Co}_{\Gamma}(s(Y))\right) \cap F(y)\right) \neq \emptyset\right.$, which, from Proposition 2.4, we have $\bigcap_{y \in Y} m-\operatorname{Cl}\left(T\left(\operatorname{Co}_{\Gamma}(s(Y))\right) \cap m-\operatorname{Cl}(F(y)) \neq \emptyset\right.$. Therefore, we have $m-\mathrm{Cl}\left(T\left(\mathrm{Co}_{\Gamma}(s(Y))\right) \cap \bigcap_{y \in Y} m-\mathrm{Cl}(F(\gamma)) \neq \emptyset\right.$. This completes the proof.

Theorem 3.6. Let $(X, D, \Gamma)$ be an abstract convex space and $Z$ be a minimal space. Suppose that $s: Y \rightarrow D$ is a mapping and $T \in m s-\operatorname{KKMC}(X, Y, Z)$ is m-compact. If a multimap $F: Y \rightarrow Z$ satisfies the following conditions.

(1) $F$ is minimal transfer closed valued,

(2) $F$ is generalized s-KKM with respect to $T$.

Then $m-\operatorname{Cl}\left(T\left(\mathrm{Co}_{\Gamma}(s(Y))\right) \cap \bigcap_{y \in Y} F(\gamma) \neq \emptyset\right.$.

Proof. It follows from Theorem 3.5 and the part (2) of Theorem 2.6.

Corollary 3.7. Let $(X, D, \Gamma)$ is an abstract convex space and $Y$ is a minimal space. Let $T \in m$-KKMC $(X, Y)$ be m-compact. If a multimap $F: D \multimap Y$ satisfies the following conditions.

(1) $F$ is minimal transfer closed valued,

(2) $F$ is generalized KKM with respect to $T$. 
Then $m-\operatorname{Cl}\left(T\left(\operatorname{Co}_{\Gamma}(D)\right)\right) \cap \bigcap_{x \in D} F(x) \neq \emptyset$.

Proof. All conditions of Theorem 3.6 are satisfied for $D$ instead of $Y$ and for $Y$ instead of $Z$, where $s$ is the identity map on $D$. Therefore, one can deduce that $m-\operatorname{Cl}\left(T\left(\operatorname{Co}_{\Gamma}(D)\right)\right) \cap \bigcap_{x \in D} F(x) \neq \emptyset$.

Remark 3.8. Note that

(1) Theorem 3.5 generalizes Theorem 4.3 in [23] in convex space, theorems 13 and 14 in [25] in L-convex spaces, theorems 3.2 and 3.3 in [26], Theorem 3.2 in [27] in $F C$-spaces, Theorem 1 in [28] in G-convex spaces and Corollary 1.1 in [29] in abstract convex minimal spaces.

(2) Theorem 3.6 generalizes Theorem 3.1 in [26] and [27], without the assumption "compactly" in $F C$-spaces and Corollary 3.1 [29] in abstract convex minimal spaces.

(3) Corollary 3.7 generalizes Theorem 2.2 in [12] from convex spaces to abstract convex spaces and also from the class $\operatorname{KKM}(X, Y)$ in convex spaces to the class $m$ $\operatorname{KKM}(X, Y)$ in abstract convex spaces.

(4) The compactness condition of multimap $T$ in Theorem 3.5 and Theorem 3.6 can be replaced by the coercivity condition (see [24]) in minimal spaces. However, we use this condition in the following theorems.

Theorem 3.9. Let $(X, D, \Gamma)$ be an abstract convex space and $Y$ be a minimal space. Let $T \in m$-KKMC $(X, Y)$ be m-compact. If $F, G, H: D \rightarrow Y$ are three multimaps satisfying the following conditions.

(1) $F$ is minimal transfer closed valued,

(2) $F^{*}(y) \subseteq G^{*}(y)$ and $H^{*}(y) \subseteq T^{*}(y)$ for any $y \in Y$,

(3) $\mathrm{Co}_{\Gamma}\left(G^{*}(y)\right) \subseteq H^{*}(y)$ for any $y \in Y$.

Then $m-\operatorname{Cl}\left(T\left(\operatorname{Co}_{\Gamma}(D)\right)\right) \cap \bigcap_{x \in D} F(x) \neq \emptyset$.

Proof. We claim that $G$ is generalized KKM with respect to $T$. To see this, suppose that there exists $A \in\langle D\rangle$ such that $T(\Gamma(A)) \nsubseteq \cup_{x \in A} G(x)$. Then there exist $\bar{x} \in \Gamma(A)$ and $\bar{y} \in T(\bar{x})$ such that $\bar{y} \notin G(x)$ for all $x \in A$. Hence $x \in G^{*}(\bar{y})$ for all $x \in A$ and so $A \subseteq G^{*}(\bar{y})$. From (3), we have $\bar{x} \in \Gamma(A) \subset H^{*}(\bar{y})$ and then the condition (2) implies that $\bar{x} \in T^{*}(\bar{y})$. Thus it follows that $\bar{y} \notin T(\bar{x})$, which is a contradiction. Therefore, $G$ is generalized KKM with respect to $T$. Since $G^{* *}(y) \subseteq F^{*}(y)$ for all $y \in Y$, from Proposition 2.1, $F$ is generalized KKM with respect to $T$. Now, all conditions of Corollary 3.7 hold and hence $m-\mathrm{Cl}\left(T\left(\mathrm{Co}_{\Gamma}(D)\right)\right) \cap \bigcap_{x \in D} F(x) \neq \emptyset$. This completes the proof.

Remark 3.10. According to the Remark 3.8, Theorem 3.9 generalizes the main theorem in [30] in FC-spaces, also it generalizes Theorem 2 in [31].

Theorem 3.11. Let $(X, D, \Gamma)$ be an abstract convex space and $Y$ be a minimal space. Let $T \in m-\operatorname{KKMC}(X, Y)$ be m-compact. If $F: D \rightarrow Y$ and $H, P: Y \rightarrow D$ are three multimaps satisfying the following conditions.

(1) $F$ is minimal transfer closed valued and $D \neq F(y)$ for all $y \in Y$,

(2) $H^{*}(x) \subseteq F(x)$ for any $x \in D$, 
(3) $\mathrm{Co}_{\Gamma}(H(y)) \subseteq P(y)$ for all $y \in Y$.

Then there exists $(\bar{x}, \bar{y}) \in D \times Y$ such that $\bar{y} \in T(\bar{x})$ and $\bar{x} \in P(\bar{y})$.

Proof. By the condition (2), it is easy to see that $F^{*}(y) \subseteq\left(H^{*}\right)^{*}(y)$ for all $y \in Y$. Now, suppose that the conclusion does not hold, that is $T(x) \cap P^{-}(x)=\emptyset$ for all $x \in D$. Choose $x \in P(y)$. Then $y \in P^{-}(x)$ and so $y \notin T(x)$, which gives $x \in T^{*}(y)$. Thus $P(y) \subset$ $T^{*}(y)$ for all $y \in Y$. The condition (3) implies that $\mathrm{Co}_{\Gamma}\left(\left(H^{*}\right)^{*}(y)\right) \subseteq\left(P^{*}\right)^{*}(y)$ for all $y \in Y$. Hence all conditions of Theorem 3.9 are satisfied for $P^{* *}$ and $H^{*}$, which implies that $m-\mathrm{Cl}(T(\Gamma(s(Y)))) \cap \bigcap_{x \in D} F(x) \neq \emptyset$ and so there exists $y_{0} \in m-\mathrm{Cl}\left(T\left(\mathrm{Co}_{\Gamma}(D)\right)\right)$ such that $y_{0} \in F(x)$ for all $x \in D$. This means that $D=F^{-}\left(y_{0}\right)$, which contradicts the condition (1). Therefore, there exists $(\bar{x}, \bar{y}) \in D \times Y$ such that $\bar{y} \in T(\bar{x})$ and $\bar{x} \in P(\bar{y})$. This completes the proof.

Theorem 3.12. Let $(X, D, \Gamma)$ be an abstract convex space and $Y$ be a minimal space. Let $T \in m$-KKMC $(X, Y)$ be $m$-compact. If $H, P, Q: Y \rightarrow D$ are three multimaps satisfying the following conditions.

(1) $Q^{-}$is minimal transfer open valued and $Q(y) \neq \emptyset$ for all $y \in Y$,

(2) $H^{*}(x) \subseteq Q^{*}(x)$ for all $x \in D$,

(3) $\mathrm{Co}_{\Gamma}(H(y)) \subseteq P(y)$ for all $y \in Y$.

Then there exists $(\bar{x}, \bar{y}) \in D \times Y$ such that $\bar{y} \in T(\bar{x})$ and $\bar{x} \in P(\bar{y})$.

Proof. The condition (1) and Theorem 2.6 imply that $Q^{*}$ is minimal transfer closed valued. By the definition of $Q^{*},\left(Q^{*}\right)^{-}=D \backslash Q(y)$ and so $D \neq\left(Q^{*}\right)^{-}(y)$ for all $y \in Y$. By applying Theorem 3.11 with $Q^{*}$ instead of $F$, there exists $(\bar{x}, \bar{y}) \in D \times Y$ such that $\bar{y} \in T(\bar{x})$ and $\bar{x} \in P(\bar{y})$. This completes the proof.

Remark 3.13. Theorem 3.12 generalizes Theorem 2.5 in [12] from convex spaces to abstract convex spaces and from the class $\operatorname{KKM}(X, Y)$ in convex spaces to the class $m$ $\operatorname{KKM}(X, Y)$ in abstract convex spaces.

Lemma 3.14. Let $(X \supseteq D, \Gamma)$ be an abstract convex space and $Y$ be a minimal space. Let $T \in m-\mathrm{KKMC}(X, Y)$. If $M$ is an abstract convex subset of $X$, then $T /{ }_{M} \in m$ - KKMC $(M, Y)$.

Proof. Set $D^{\prime}=D \cap M$ and let $\Gamma^{\prime}:\left\langle D^{\prime}\right\rangle \rightarrow M$ be the multimap defined by $\Gamma^{\prime}(A)=\Gamma(A)$ for any $A \in\left\langle D^{\prime}\right\rangle$. Consider a multimap $F: M \multimap Y$ defined by $F(x)=m-\mathrm{Cl}\left(A_{x}\right)$ for all $x$ $\in M$. Then $F$ is generalized KKM with respect to $\left.T\right|_{M}$. Now, define the multimap $G$ : $D \multimap Y$ by

$$
G(x)= \begin{cases}F(x), & \text { if } x \in D^{\prime} \\ Y, & \text { if } x \in D \backslash D^{\prime}\end{cases}
$$

It is not hard to check that $G$ is generalized KKM with respect to $T$. Since $T \in m$ $\operatorname{KKMC}(X, Y)$, the family $\{G(x): x \in D\}$ has the finite intersection property and so $\{F(x)$ : $\left.x \in D^{\prime}\right\}$ has the finite intersection property. Then $\left.T\right|_{M} \in m-\operatorname{KKMC}(M, Y)$. This completes the proof.

In the results on the KKM and coincidence type theorems mentioned above, it is assumed that $T \in m s-\operatorname{KKMC}(X, Y, Z)$ or $T \in m$ - $\operatorname{KKMC}(X, Y)$ is an $m$-compact 
multimap, but, when it is not $m$-compact, we can use the coercivity conditions instead of the $m$-compactness condition of $T$ as follows.

Proposition 3.15[14]. Let $X$ and $Y$ be two minimal spaces. Then the following statements for a multimap $F: X \rightarrow Y$ are equivalent.

(1) $F^{*}: Y \rightarrow X$ is minimal transfer open valued and $F(x) \neq \emptyset$ for all $x \in X$.

(2) $X=\mathrm{U}\left\{m\right.$-Int $\left.\left(F^{-}(y)\right): y \in Y\right\}$.

Definition 3.16[32]. Let $(X, \mathcal{M})$ be a minimal space and $Y$ be a nonempty subset of $X$. The family $\left.\mathcal{M}\right|_{Y}=\{U \cap Y: U \in \mathcal{M}\}$ is called induced minimal structure by $\mathcal{M}$ on $Y$. $\left(Y,\left.\mathcal{M}\right|_{Y}\right)$ is called minimal subspace of $(X, \mathcal{M})$. Also, for any subset $A$ of $X$ we define $m-\operatorname{Int}_{Y}(A)=\cup\left\{V:\left.V \in \mathcal{M}\right|_{Y}\right.$ and $\left.V \subseteq A\right\}$.

Lemma 3.17. Let $(X, \mathcal{M})$ be a minimal space and $A$ be a nonempty subset of $X$. Then we have

$$
m-\operatorname{Int}(\mathrm{A}) \cap B \subseteq m-\operatorname{Int}_{B}(A \cap B) .
$$

Proof. From Definition 3.16, we have

$$
\begin{aligned}
m-\text { Int }(A) \cap B & =\bigcup\{U: U \in \mathcal{M} \text { and } U \subseteq A\} \cap B \\
& =\bigcup\{U \cap B: U \in \mathcal{M} \text { and } U \subseteq A\} \\
& \subseteq \bigcup\left\{G:\left.G \in \mathcal{M}\right|_{B} \text { and } G \subseteq A \cap B\right\} \\
& =m-\operatorname{Int}_{B}(A \cap B) .
\end{aligned}
$$

Theorem 3.18. Let $(X \supseteq D, \Gamma)$ be an abstract convex space, $Y$ be a minimal space and $T \in m-\operatorname{KKMC}(X, Y)$. If $H, P, Q: Y \rightarrow D$ are three multimaps satisfying the following conditions.

(1) $Q^{-}$is minimal transfer open valued and $Q(\gamma) \neq \emptyset$ for all $y \in Y$,

(2) $H^{*}(x) \subseteq Q^{*}(x)$ for all $x \in D$,

(3) $\mathrm{Co}_{\Gamma}(H(y)) \subseteq P(y)$ for all $y \in Y$,

(4) for each m-compact subset $C \subseteq D, m-\mathrm{Cl}(T(C))$ is m-compact in $Y$,

(5) there is an m-compact subset $K \subseteq Y$ such that, for any $A \in\langle D\rangle$, there is an $m$ compact abstract convex subset $L_{A} \subseteq D$ containing $A$ such that $T\left(L_{A}\right) \backslash K \subseteq \cup_{x \in L_{A}} m-\operatorname{Int}(Q-(x))$.

Then there exists $(\bar{x}, \bar{y}) \in D \times Y$ such that $\bar{y} \in T(\bar{x})$ and $\bar{x} \in P(\bar{y})$.

Proof. The condition (1) and Proposition 3.15 imply that $Y=\cup_{x \in D} m-\operatorname{Int}\left(Q^{-}(x)\right)$ and so we have

$$
K=\bigcup_{x \in D} m-\operatorname{Int}\left(Q^{-}(x)\right) \cap K \subseteq \bigcup_{x \in D} m-\operatorname{Int}\left(Q^{-}(x)\right)=\bigcup_{x \in D} \bigcup_{j \in J_{x}} U_{x, j}
$$

where $U_{x, j}$ is $m$-open and $U_{x, j} \subseteq Q^{-}(x)$. Since $K$ is an $m$-compact subset of $Y$, $K \subseteq \cup_{i=1}^{n} U_{x_{i} j_{i}} \subseteq \cup_{i=1}^{n} m-\operatorname{Int}\left(Q^{-}\left(x_{i}\right)\right)$ - for $j_{i} \in J_{x_{i}}$.

On the other hand, by the condition (5), there exists an $m$-compact abstract convex subset $L_{A}$ of $D$ containing $A$ such that $T\left(L_{A}\right) \backslash K \subseteq \cup_{x \in L_{A}} m-\operatorname{Int}\left(Q^{-}(x)\right)$. Thus we have 


$$
T\left(L_{A}\right) \subseteq \bigcup_{x \in L_{A}} m-\operatorname{Int}\left(Q^{-}(x)\right) \cup K=\bigcup_{x \in L_{A}} m-\operatorname{Int}\left(Q^{-}(x)\right) \cup \bigcup_{i=1}^{n} m-\operatorname{Int}\left(Q^{-}\left(x_{i}\right)\right),
$$

where $x_{i} \in A \subseteq L_{A}$. Then $T\left(L_{A}\right) \subseteq \cup_{x \in L_{A}} m-\operatorname{Int}\left(Q^{-}(x)\right)$. By Lemma 3.17, it follows that

$$
T\left(L_{A}\right)=\bigcup_{x \in L_{A}} m-\operatorname{Int}\left(Q^{-}(x)\right) \cap T\left(L_{A}\right) \subseteq \bigcup_{x \in L_{A}} m-\operatorname{Int}_{T}\left(L_{A}\right)\left(Q^{-}(x) \cap T\left(L_{A}\right)\right) \subseteq T\left(L_{A}\right) .
$$

From the condition (4), it follows that $m-\mathrm{Cl}\left(T\left(L_{A}\right)\right)$ is $m$-compact subset of $Y$. Moreover, since $L_{A}$ is an abstract convex subset of $X$, applying Lemma 3.14, $\left.T\right|_{L_{A}} \in m-\operatorname{KKMC}\left(L_{A}, Y\right)$.

Now, consider three multimaps $\bar{P}, \bar{Q}, \bar{H}: T\left(L_{A}\right) \multimap L_{A}$ defined by $\bar{P}(\gamma)=P(y) \cap L_{A}$, $\bar{Q}(\gamma)=Q(\gamma) \cap L_{A}$ and $\bar{H}(\gamma)=H(\gamma) \cap L_{A}$ for all $y \in T\left(L_{A}\right)$. Then $\left.T\right|_{L_{A}}, \bar{P}, \bar{Q}$ and $\bar{H}$ satisfy in all conditions of Theorem 3.12 (notice Proposition 3.15) and hence there exist $\bar{y} \in T\left(L_{A}\right) \subseteq Y \quad$ and $\quad \bar{x} \in L_{A} \subseteq D$ such that $\left.\bar{y} \in T\right|_{L_{A}}(\bar{x})=T(\bar{x})$ and $\bar{x} \in \bar{P}(\bar{y}) \subseteq P(\bar{y})$. This completes the proof.

Corollary 3.19. Let $(X \supseteq D, \Gamma)$ be an abstract convex space, $Y$ be a minimal space and $T \in m-\operatorname{KKMC}(X, Y)$. If $P, Q: Y \rightarrow D$ are two multimaps satisfying the following conditions.

(1) $Q^{-}$is minimal transfer open valued and $Q(\gamma) \neq \emptyset$ for all $y \in Y$,

(2) $\mathrm{Co}_{\Gamma}(Q(y)) \subseteq P(y)$ for all $y \in Y$,

(3) for each m-compact subset $C \subseteq D, m-\mathrm{Cl}(T(C))$ is m-compact in $Y$,

(4) there exists an m-compact subset $K \subseteq Y$ such that, for any $A \in\langle D\rangle$, there exists an m-compact abstract convex subset $L_{A} \subseteq D$ containing $A$ satisfying $T\left(L_{A}\right) \backslash K \subseteq \cup_{x \in L_{A}} m-\operatorname{Int}\left(Q^{-}(x)\right)$.

Then there exists $(\bar{x}, \bar{y}) \in D \times Y$ such that $\bar{y} \in T(\bar{x})$ and $\bar{x} \in P(\bar{y})$.

Proof. Letting $H^{*}=Q^{*}$ in Theorem 3.18, the conclusion follows.

Remark 3.20. Theorem 3.18 and Corollary 3.19 generalize Theorem 2.6 in [12] from the class of convex spaces to the class of abstract convex spaces under the weaker assumptions.

\section{Applications to minimax inequalities}

Fan's minimax inequality [33] has played very important roles in the study of modern nonlinear analysis and especially in mathematical economics. Moreover, some general minimax theorems and extensions of these inequalities have been obtained for the functions with KKM property under the various weaker conditions in many spaces with the convex structure (see [10] and [34-39]).

In this section, we prove some results to obtain Ky Fan's type minimax theorem as follows.

Theorem 4.1. Let $(X \supseteq D, \Gamma)$ be an abstract convex space, $Y$ be a minimal space and $T: X \rightarrow Y$ is a multimap and $T \in m-\operatorname{KKMC}(X, Y)$. If $G, H: D \multimap Y$ are two multimaps satisfying the following conditions. 
(1) $G^{*}(y) \subseteq T^{*}(y)$ for all $y \in Y$,

(2) $\mathrm{Co}_{\Gamma}\left(H^{*}(y)\right) \subseteq G^{*}(y)$ for all $y \in Y$,

(3) $H$ is minimal transfer closed valued,

(4) for any m-compact subset $C \subseteq D, m-\mathrm{Cl}(T(C))$ is m-compact in $Y$,

(5) there exists an m-compact subset $K \subseteq Y$ such that, for any $A \in\langle D\rangle$, there exists an $m$-compact abstract convex subset $L_{A} \subseteq D$ containing $A$ satisfying $T\left(L_{A}\right) \cap \bigcap_{x \in L_{A}} m-\mathrm{Cl}(H(x)) \subseteq K$.

Then $\bigcap_{x \in D} H(x) \neq \emptyset$.

Proof. Suppose that $\bigcap_{x \in D} H(x)=\emptyset$. For all $y \in Y$, there exists $x \in D$ such that $y \notin$ $H(x)$ and so $x \in H^{*}(y)$. Thus $H^{*}$ is nonempty valued. Since $H$ is minimal transfer closed valued, from Lemma 2.6 and Proposition 2.1, it follows that $H^{c}=\left(H^{*}\right)^{-}$is minimal transfer open valued and $m-\mathrm{Cl}(H(x))=\left(m-\operatorname{Int}\left(\left(H^{*}\right)^{-}(x)\right)\right)^{c}$ for all $x \in D$. Hence, from the condition (5), there exists an $m$-compact subset $K \subseteq Y$ such that, for any $A \in$ $\langle D\rangle$, there exists an $m$-compact abstract convex subset $L_{A} \subseteq D$ containing $A$ such that $\left.T\left(L_{A}\right) \backslash K \subseteq \cup_{x \in L_{A}} m-\operatorname{Int}\left(H^{*}\right)^{-}(x)\right)$. Applying Corollary 3.19 for $\left(G^{*}, H^{*}\right)$ instead of $(P$, $Q)$, there exists $(\bar{x}, \bar{y}) \in D \times Y$ such that $\bar{y} \in T(\bar{x})$ and $\bar{x} \in G^{*}(\bar{y})$. Thus $\bar{x} \notin T^{*}(\bar{y})$ and $\bar{x} \in G^{*}(\bar{y})$, which contradicts the condition (1). Therefore, $\bigcap_{x \in D} H(x) \neq \emptyset$.

Corollary 4.2. Let $(X \supseteq D, \Gamma)$ be an abstract convex space, $Y$ be a minimal space and $T \in m-\mathrm{KKMC}(X, Y)$. If $H: D \rightarrow Y$ is a multimap satisfying the following conditions.

(1) $\mathrm{Co}_{\Gamma}\left(H^{*}(y)\right) \subseteq T^{*}(y)$ for all $y \in Y$,

(2) $H$ is minimal transfer closed valued,

(3) for any $m$-compact subset $C \subseteq D, m-\mathrm{Cl}(T(C))$ is $m$-compact in $Y$,

(4) there exists an m-compact subset $K \subseteq Y$ such that, for any $A \in\langle D\rangle$, there exists an m-compact abstract convex subset $L_{A} \subseteq D$ containing $A$ satisfying $T\left(L_{A}\right) \cap \bigcap_{x \in L_{A}} m-\mathrm{Cl}(H(x)) \subseteq K$.

Then $\bigcap_{x \in D} H(x) \neq \emptyset$.

Proof. Letting $\mathrm{G}=\left.\mathrm{T}\right|_{D}$ in Theorem 4.1, we can get the conclusion.

Lemma 4.3. Let $(X, \Gamma)$ is an abstract convex space and $Y$ be a nonempty set. If $T, S$ : $X \rightarrow Y$ are two multimaps, then the following statements are equivalent.

(1) $\mathrm{Co}_{\Gamma}\left(T^{*}(y)\right) \subseteq S^{*}(y)$ for each $y \in Y$.

(2) For any $A \in\langle X\rangle, S(\Gamma(A)) \subseteq T(A)$.

Proof. (1) $\Rightarrow(2)$ : Let $A \in\langle X\rangle$ and $y \in S(\Gamma(A))$. Then there exists $x \in \Gamma(A)$ such that $y$ $\in S(x)$. Hence, from the definition of $S^{-}, x \in \Gamma(A) \cap S^{-}(y) \neq \emptyset$. So, it follows from Proposition 2.1 that $\Gamma(A) \nsubseteq S^{*}(y)$. From (1), it follows that $A \nsubseteq T^{*}(y)$ and so $A \cap T^{-}(y) \neq \emptyset$. Choose $z \in A \cap T^{-}(y)$. Then $y \in T(z) \subseteq T(A)$. This means that $S(\Gamma(A)) \subseteq$ $T(A)$ and so $(1) \Rightarrow(2)$ is proved.

(2) $\Rightarrow(1)$ : Choose $y \in Y$ such that $\cup_{A \in\left\langle T^{*}(y)\right\rangle} \Gamma(A)=\mathrm{Co}_{\Gamma}\left(T^{*}(y)\right) \nsubseteq S^{*}(y)$. Then there exist $A \in\left\langle T^{*}(y)\right\rangle$ and $x \in \Gamma(A)$ such that $x \notin S^{*}(y)$, which implies that $y \in S(x) \subseteq S$ $(\Gamma(A))$. On the other hand, $A \in\left\langle T^{*}(y)\right\rangle$ implies that $A \subseteq D \backslash T^{(}(y)$ and so 
$A \cap T^{-}(y)=\emptyset$. Thus it follows that $\mathrm{y} \notin T(A)$ and hence $S(\Gamma(A)) \nsubseteq T(A)$, which this contradicts (2).

Remark 4.4. Note that

(1) If $X=D$, then, by Lemma 4.3, in the condition (2) of Theorem 4.1, we can put $G(\Gamma(A)) \subseteq H(A)$ for all $A \in\langle D\rangle$ and so $T(\Gamma(A)) \subseteq H(A)$ for all $A \in\langle D\rangle$ instead of the condition (1) in Corollary 4.2.

(2) Theorem 4.1 and Corollary 4.2 are generalizations of Theorem 3.3 and Corollary 3.2 in [40] respectively.

Definition 4.5. Let $(X \supseteq D, \Gamma)$ be an abstract convex space, $Y$ be a nonempty set and $f$ be a real-valued bifunction defined on $X \times Y$. Then $f$ is said to be

(1) quasi-abstract convex in the first variable if, for all $y \in Y$ and $\gamma \in \mathbb{R}$, the set ( $x$ $\in X: f(x, y)<\gamma\}$ is abstract convex.

(2) quasi-abstract concave in the first variable if, for all $y \in Y$ and $\gamma \in \mathbb{R}$, the set $(x$ $\in X: f(x, y)>\gamma\}$ is abstract convex.

Definition 4.6. Let $X, Y$ be minimal spaces and $f$ be a real-valued bifunction defined on $X \times Y$. Then $f$ is said to be

(1) strongly path minimal transfer lower semi-continuous in the first variable (shortly, spmt l.s.c.) if, for any $(x, y) \in X \times Y$ and $\varepsilon>0$, there exists an $m$-open set $N(x)$ containing $x$ in $X$ and there exists $y^{\prime} \in Y$ such that $\mathrm{f}(\mathrm{x}, \mathrm{y})<f\left(x^{\prime}, y^{\prime}\right)+\varepsilon$ for any $x^{\prime} \in N(x)$.

(2) strongly path minimal transfer upper semi-continuous in the first variable (shortly, spmt u.s.c.) if the function - $f$ is l.s.c. in the first variable.

Theorem 4.7. Let $(X \supseteq D, \Gamma)$ be an abstract convex space, $Y$ be a minimal space and $T \in m-\operatorname{KKMC}(X, Y)$. If $f, g: D \times Y \rightarrow \mathbb{R}$ are two functions and $\gamma=\inf _{(x, y) \in G_{T}} f(x, y)$ satisfying the following conditions.

(1) for any m-compact subset $C \subseteq D, m-\mathrm{Cl}(T(C))$ is m-compact in $Y$,

(2) $f(x, y) \leq g(x, y)$ for all $(x, y) \in D \times Y$,

(3) $f$ is quasi-abstract convex in the first variable,

(4) $g$ is spmt u.s.c in the second variable,

(5) there exists an m-compact subset $K \subseteq Y$ such that, for any $A \in\langle D\rangle$, there exists an m-compact abstract convex subset $L_{A} \subseteq D$ containing $A$ such that, for all $\bar{y} \in T\left(L_{A}\right) \backslash K$, there exists $x \in L_{A}$ such that $\bar{y} \in m-\operatorname{Int}\{y: g(x, y)<\gamma\}$. Then

$$
\inf _{(x, y) \in G_{T}} f(x, y) \leq \sup _{y \in Y} \inf _{x \in D} g(x, y) .
$$

Proof. Consider the multimap $H: D \multimap Y$ defined by

$$
H(x)=\{y \in Y: g(x, y) \geq \gamma\}
$$

for all $x \in D$. We claim that $\mathrm{Co}_{\Gamma}\left(H^{*}(\bar{y})\right) \subseteq T^{*}(\bar{y})$ for all $y \in Y$. Suppose that this is not the case, so there exists $\bar{y} \in Y$ such that $\operatorname{Co}_{\Gamma}\left(H^{*}(\bar{y})\right) \nsubseteq T^{*}(\bar{y})$ or 
$\bar{x} \notin X \backslash T^{-}(\bar{y})$.. Thus there exist $A \in\left\langle D \backslash H^{-}(\bar{y})\right\rangle$ and $\bar{x} \in \Gamma(A)$ such that $\bar{x} \notin X \backslash T^{-}(\bar{y})$. Then $\bar{y} \in T(\bar{x})$, which implies that $(\bar{x}, \bar{y}) \in G_{T}$.

On the other hand, from $A \subseteq D \backslash H^{-}(\bar{y})$, we have $A \cap H^{-}(\bar{\gamma})=\emptyset$. It follows that $\bar{y} \notin H(A)$ and hence $g(x, \bar{y})<\gamma$, for all $x \in A$. By the condition (2), we have $f(x, \bar{y})<\gamma$ for all $x \in A$. The condition (3) implies that $f(x, \bar{y})<\gamma$ for all $x \in \Gamma(A)$. Therefore, $f(\bar{x}, \bar{y})<\gamma$, which contradicts $\gamma=\inf _{(x, y) \in \mathrm{G}_{T}} f(x, y)$. Thus it follows that $\mathrm{Co}_{\Gamma}\left(H^{*}(y)\right) \subseteq T^{*}(y)$ for all $y \in Y$.

To prove that $H$ is minimal transfer closed valued, suppose that $(x, y) \in D \times Y$ and $y$ $\notin H(x)$ and hence $g(x, y)<\gamma$. By the condition (4), setting $\varepsilon=\gamma-g(x, y)$, there exist $x^{\prime} \in$ $D$ and an $m$-open set $N(y)$ containing $y$ such that

$$
-g(x, y)<-g\left(x^{\prime}, \gamma^{\prime}\right)+\varepsilon=-g\left(x^{\prime}, y^{\prime}\right)+\gamma-g(x, y)
$$

for all $y^{\prime} \in N(y)$. Thus $g\left(x^{\prime}, y^{\prime}\right)<\gamma$ for all $y^{\prime} \in N(y)$ and so $N(y) \cap H\left(x^{\prime}\right)=\emptyset$. It follows that $N(y) \subseteq\left(H\left(x^{\prime}\right)\right)^{c}$ and so we have

$$
y \in N(y) \subseteq m-\operatorname{Int}\left(\left(H\left(x^{\prime}\right)\right)^{c}\right)=\left(m-\operatorname{Cl}\left(H\left(x^{\prime}\right)\right)\right)^{c} .
$$

Therefore, $y \notin m-\mathrm{Cl}\left(H\left(x^{\prime}\right)\right)$. From the condition (5), it follows that $T\left(L_{A}\right) \backslash K \subseteq \cup_{x \in L_{A}} m-\operatorname{Int}\left(H^{c}(x)\right)$, which implies that $T\left(L_{A}\right) \backslash K \subseteq\left(\cap_{x \in L_{A}} m-\mathrm{Cl}\left(H^{c}(x)\right)\right.$ or $T\left(L_{A}\right) \cap \cap_{x \in L_{A}} m-\mathrm{Cl}(H(x)) \subseteq K$. Thus the condition (5) is equivalent to the condition (4) in Corollary 4.2 for multimap $H$.

Now, applying Corollary 4.2, we have $\bigcap_{x \in D} H(x) \neq \emptyset$. There exists $y^{\prime} \in Y$ such that $g(x$, $\left.y^{\prime}\right) \geq \gamma$ for all $x \in D$ and so $\sup _{y \in Y} \inf _{x \in D} g(x, y) \geq \gamma$. This implies that

$$
\inf _{(x, y) \in G_{T}} f(x, y) \leq \sup _{y \in Y} \inf _{x \in D} g(x, y) .
$$

Remark 4.8. Note that

(1) The conditions (2) and (3) in Theorem 4.7 are satisfied if we assume that $f$ is $g$ quasiconvex in the first variable, that is, for any $y \in Y$ and $A \in\langle D\rangle$, we have $f(x, y) \leq \max _{x \in A} g(x, y)$ for all $x \in \Gamma(A)$.

(2) Theorem 4.7 is a generalization of Theorem 1 in [41], Theorem 8 in [39] and Theorem 6.4 in [42].

\section{Acknowledgements}

The first author was supported by the Basic Science Research Program through the National Research Foundation of Korea (NRF) funded by the Ministry of Education, Science and Technology (Grant Number: 2011-0021821).

\footnotetext{
Author details

${ }^{1}$ Department of Mathematics Education and the RINS Gyeonsang National University, Chinju 660-701, Korea

${ }^{2}$ Department of Mathematics, Faculty of Sciences, Semnan University, P. O. BOX 35195-363, Semnan, Iran ${ }^{3}$ Islamic Azad University-Babol Branch, Babol, Iran ${ }^{4}$ Department of Mathematics, Faculty of Sciences Golestan University, P.O.Box. 155, Gorgan, Iran
}

Authors' contributions

All authors read and approved the final manuscript.

Competing interests

The authors declare that they have no competing interests. 


\section{References}

1. Knaster, B, Kuratowski, K, Mazurkiewicz, S: Ein beweis des fixpunktsatsez für $n$-dimensionale simplexe. Fund Math. 14, 132-137 1 (1929)

2. Park, S: Foundations of the KKM theory via coincidences of composites of upper semicontinuous maps. J Korean Math Soc. 31, 493-519 1 (1994)

3. Park, S: Some coincidence theorems on acyclic multifunctions and applications to KKM theory. In: Tan K-K (ed.) Fixed Point Theory and Applications. pp. 248-277. World Scientific Publishers, River Edge (1992). 1

4. Lassonde, M: On the use of KKM multifunctions in fixed point theory and related topics. J Math Anal Appl. 97, 151-201 1 (1983). doi:10.1016/0022-247X(83)90244-5

5. Horvath, CD: Contractibility and generalized convexity. J Math Anal Appl. 156, 341-357 (1991). doi:10.1016/0022-247X (91)90402-L

6. Horvath, CD: Extension and selection theorems in topological spaces with a generalized convexity structure. Ann Fac Sci Toulouse Math. 6, 253-269 (1993)

7. Horvath, CD: Convexite generalise et applications. In Sem Math Super, vol. 110, pp. 79-99.Press, University of Montre'al (1990)

8. Horvath, CD: Some results on multivalued mappings and inequalities without convexity. In: Lin BL, Simons $S$ (eds.) Nonlinear and Convex Analysis (Proc. in honor of Ky Fan). pp. 99-106. Marcel Dekker, New York (1987)

9. Park, S, Kim, H: Admissible classes of multifunctions on generalized convex spaces. Proc Coll Nature Sci SNU 18, 1-21 (1993). 1, 2

10. Park, S: Coincidence, almost fixed point, and minimax theorems on generalized convex spaces. J Nonlinear Convex Anal. 4, 151-164 (2003)

11. Chang, TH, Yen, CL: KKM property and fixed point theorems. J Math Anal Appl 203, 224-235 (1996). 1. doi:10.1006/ jmaa.1996.0376

12. Lin, $\sqcup$, Ansari, $\mathrm{OH}, \mathrm{Wu}, J Y$ : Geometric properties and coincidence theorems whit applications to generalized vector equilibrium problems. J Optim Theory Appl 117, 121-137 (2003). 1, 3.8, 3.13, 3.20. doi:10.1023/A:1023656507786

13. Alimohammady, M, Roohi, M, Delavar, MR: Knaster-Kuratowski-Mazurkiewicz theorem in minimal generalized convex spaces. Nonlinear Funct Anal Appl 13, 483-492 (2008). 2, 2.3, 2

14. Alimohammady, M, Roohi, M, Delavar, MR: Transfer closed and transfer open multimaps in minimal spaces. Chaos, Solitons Fractals 40, 1162-1168 (2009). 2.5, 2, 3.15. doi:10.1016/j.chaos.2007.08.071

15. Alimohammady, M, Roohi, M, Delavar, MR: Transfer closed multimaps and Fan-KKM principle. Nonlinear Funct Anal Appl 13, 597-611 (2008). 2, 2.9

16. Ding, XP: Maximal element theorems in product FC-spaces and generalized games. J Math Anal Appl 305, 29-42 (2005). 1. doi:10.1016/j.jmaa.2004.10.060

17. Horvath, CD, Llinares Ciscar, JV: Maximal elements and fixed point for binary relations on topological ordered spaces. J Math Econ 25, 291-306 (1996). 1. doi:10.1016/0304-4068(95)00732-6

18. Zafarani, J: KKM property in topological spaces. Bull Soc R Liege 73, 171-185 (2004). 1

19. Park, S: On generalizations of the KKM principle on abstract convex spaces. Nonlinear Anal Forum 11, 67-77 (2006). 1, 3.1

20. Lan, KQ, Wu, JH: A fixed-point theorem and applications to problems on sets with convex sections and to Nash equilibria. Math Comput Model 36, 139-145 (2002). 2.1. doi:10.1016/S0895-7177(02)00110-3

21. Maki, H: On generalizing semi-open sets and preopen sets. Proceedings of the 8 th Meetings on Topolgical Spaces Theory and its Application 13-18 (1996). 2, 2.4

22. Popa, V, Noiri, T: On M-continuous functions. Anal. Univ. "Dunarea Jos-Galati". Ser Mat Fiz Mec Teor Fasc II 18(23), 31-41 (2000). 2.2, 2, 2.7

23. Chang, TH, Huang, YY, Jeng, JC, Kuo, KW: On S-KKM property and related topics. J Math Anal Appl 229, 212-227 (1999). 3, 3.8. doi:10.1006/jmaa.1998.6154

24. Park, S: The KKM principle in abstract convex spaces: Equivalent formulations and applications. Nonlinear Anal 73, 1028-1042 (2010). 3.3, 3.8. doi:10.1016/j.na.2010.04.029

25. Chen, CM: R-KKM theorems on L-convex spaces and its applications. Sci Math Jpn 65, 195-207 (2007). 3.8

26. Ding, XP: Generalized KKM type theorems in FC-spaces with applications (I). J Glob Optim 36, $581-596$ (2006). 3.8 doi:10.1007/s10898-006-9028-X

27. Ding, XP, Ding, TM: KKM type theorems and generalized vector equilibrium problems in noncompact FC-spaces. J Math Anal Appl 331, 1230-1245 (2007). 3.8. doi:10.1016/j.jmaa.2006.09.059

28. Lin, LJ: A KKM type theorem and it's applications. Bull Austral Math Soc 59, 481-493 (1999). 3.8. doi:10.1017/ S0004972700033189

29. Park, S: Application of the KKM principle on abstract convex minimal space. Nonlinear Funct Anal Appl 13, 179-191 (2008). 3.8

30. Ding, XP: Generalized KKM type theorems in FC-spaces with applications (II). J Glob Optim 38, 367-385 (2007). 3.10. doi:10.1007/s10898-006-9070-8

31. Park, S: Elements of the KKM theory on abstract convex spaces. J Korean Math Soc 45, 1-27 (2008), 3.10

32. Alimohammady, M, Roohi, M: Linear minimal space. Chaos, Solitons Fractals 33, 1348-1354 (2007). 3.16. doi:10.1016/j. chaos.2006.01.100

33. Fan, K: A minimax inequality and applications. In: Sisha O (ed.) Inequalities. pp. 103-113. Academic Press, San Diego, III: (1972). 4

34. Ansari, QH, Lin, YC, Yao, JC: General KKM theorem with applications to minimax and variational inequalities. J Optim Theory Appl. 104, 41-57 (2000)

35. Chang, SS, Lee, BS, Wu, X, Cho, YJ, Lee, M: The generalized quase-variational inequalities problems. J Math Anal Appl. 203, 686-711 (1996). doi:10.1006/jmaa.1996.0406 
36. Kuo, TY, Jeng, JC, Huang, YY: Fixed point theorems for compact multimaps on almost $\Gamma$-convex sets in generalized convex spaces. Nonlinear Anal. 66, 415-426 (2007). doi:10.1016/j.na.2005.11.036

37. Lan, KQ: New fixed-point theorems for two maps and applications to problems on sets with convex section and minimax inequalities. Comput Math. 47, 195-205 (2004)

38. Lin, L: Applications of a fixed point theorem in G-convex spaces. Nonlinear Anal. 46, 601-608 (2001). doi:10.1016/ S0362-546X(99)00456-3

39. Zhang, JH: Some minimax inequalities for mappping with noncompact domain. Appl Math Lett 17, 717-720 (2003). 4.8

40. Lin, L, Wan, WP: KKM type theorems and coincidence theorems with applications to the existence of equilibria. J Optim Theory Appl 123, 105-122 (2004). 4.4

41. Ha, CW: On a minimax of Ky Fan. Proc Amer Math Soc 99, 680-682 (1987). 4.8. doi:10.1090/50002-9939-1987-0877039-9

42. Park, S: Equilibrium existence theorems in KKM spaces. Nonlinear Anal 69, 4352-5364 (2008). 4.8. doi:10.1016/j. na.2007.10.058

doi:10.1186/1029-242X-2011-126

Cite this article as: Je Cho et al:: Coincidence theorems and minimax inequalities in abstract convex spaces. Journal of Inequalities and Applications 2011 2011:126.

\section{Submit your manuscript to a SpringerOpen ${ }^{\odot}$} journal and benefit from:

- Convenient online submission

- Rigorous peer review

- Immediate publication on acceptance

- Open access: articles freely available online

- High visibility within the field

- Retaining the copyright to your article

Submit your next manuscript at $\gg$ springeropen.com 